\title{
BMJ Open Effectiveness of a comprehensive care protocol in patients with new diagnoses of type 2 diabetes mellitus and associated comorbidities in primary care: study protocol of a quasi- experimental trial
}

To cite: Lapena C, Borràs $\mathrm{E}$, Digon C, et al. Effectiveness of a comprehensive care protocol in patients with new diagnoses of type 2 diabetes mellitus and associated comorbidities in primary care: study protocol of a quasiexperimental trial. BMJ Open 2020;10:e033725. doi:10.1136/ bmjopen-2019-033725

- Prepublication history and additional material for this paper are available online. To view these files, please visit the journal online (http://dx.doi org/10.1136/bmjopen-2019033725).

Received 19 August 2019 Revised 22 April 2020 Accepted 01 May 2020

Check for updates

(C) Author(s) (or their employer(s)) 2020. Re-use permitted under CC BY-NC. No commercial re-use. See rights and permissions. Published by BMJ.

For numbered affiliations see end of article.

Correspondence to Mrs Carolina Lapena; clapena@gencat.cat

\section{ABSTRACT}

Introduction Type 2 diabetes mellitus (T2DM) is a highly prevalent chronic disease in the Spanish population. Typically, T2DM is associated with other chronic conditions. Intensive medication at the time of diagnosis has proven effective in reducing cardiovascular risk, improving glycaemic control and preventing T2DM complications. However, it has not yet been demonstrated that a comprehensive and intensive health education protocol at the time of diagnosis has the benefits described previously. Currently, there is great variability in the practices of primary care nurses regarding health education at the time of disease diagnosis.

We aimed to evaluate the effectiveness of a systematic protocol with a comprehensive care programme in people with newly diagnosed T2DM with associated comorbidities. Methods and analysis A multicentre quasi-experimental design comparing a group of individuals taking part in the intervention (intervention group (IG)) with a similar group receiving standard diabetes care (comparison group (CG)) is planned. The intervention will take place during the 3 months after study enrolment. Data will be collected at baseline and at 3, 6 and 12 months. Ten primary care centres in Barcelona city will be selected for participation: 5 for the IG and 5 for the CG. The IG will include five structured individual visits postdiagnosis with the primary care nurse, during which aspects of diabetes education will be discussed with the patient and his/her family. The results will be measured in terms of health-related quality of life and the change in main outcomes (glycated haemoglobin and weight).

Ethics and dissemination The study fully met the requirements of the Ethical Committee of Clinical Investigation of the IDIAP Jordi Gol (approval code: $\mathrm{P} 13 / 118)$. Patients will be informed that their data are confidential, and they have the right to withdraw at any time without penalty. Dissemination will include publishing the findings in peer-reviewed journals and sharing our findings at scientific conferences.

Trial registration number NCT03990857; Pre-results.
Strengths and limitations of this study

- Separation between with the intervention and standard care centres is planned to minimise the risk of contamination.

- The support materials follow the recommendations of the guidelines, were reviewed by healthcare professionals for content accuracy and were evaluated by patients for their full understanding.

- The absence of participant randomisation is a limitation.

- The questionnaire used to measure health-related quality of life is not specific to people with diabetes.

\section{INTRODUCTION}

Type 2 diabetes mellitus (T2DM) comprises more than $90 \%$ of people with diabetes worldwide. It was estimated that in 2017, there were 451 million (age 18-99 years) people with diabetes worldwide. Projections indicate that this figure will increase over the years to approximately 693 million by 2045. In the Spanish population, almost $30 \%$ of individuals have some type of disturbance of glycaemic status (dysglycaemia), and $13.8 \%$ have diabetes, of which approximately $50 \%$ had unknown diabetes. ${ }^{23}$ Additionally, chronic diabetes complications have an important impact on the quality of life of patients and a high human cost, both from the social and the economic perspectives. ${ }^{124}$

The care of people with diabetes in primary care poses a major challenge due to their multiple comorbidities that demand continuous efforts to make appropriate recommendations for self-management and medication in a stepwise manner to achieve 
evidence-based targets. The three core pillars of T2DM management and prevention of its complications are drug treatment, changes in lifestyle and education. In this sense, an intensive approach to the disease, especially at the time of diagnosis, can contribute to the prevention of both microangiopathic complications and cardiovascular events, regardless of the degree of glycaemic control in more advanced stages. ${ }^{5}$ It is uncertain, however, whether intensive control of the disease in more advanced stages, particularly in older patients with coexisting chronic complications or significant comorbidity, has benefits in terms of macrovascular complications and global mortality. ${ }^{6} 7$

The benefits of controlling T2DM and its accompanying cardiovascular risk factors are well established in clinical practice guidelines. ${ }^{8-11}$ Current guidelines for the prevention and management of T2DM point to the need to implement changes in lifestyle, such as diet and exercise, as crucial elements of treatment ${ }^{12}{ }^{13}$; however, a major focus of drug treatment (including multiple combinations) is to reduce blood glucose and the associated elevated risks of cardiovascular disease. ${ }^{1415}$ Evidence from cross-sectional studies indicates that there are barriers and difficulties in achieving these treatment objectives. ${ }^{16}{ }^{17}$ However, some studies have also shown the positive changes in main outcomes. ${ }^{18-20}$ Previous studies have also shown the benefits of glycaemic control on microvascular risk if drug intensification occurs at the onset of T2DM. ${ }^{21}$ Furthermore, lifestyle interventions, that is, increasing physical activity, reducing caloric intake and education regarding treatment adherence, have also demonstrated a reduction of new T2DM diagnoses, a reduction of glycaemic control (HbA1c) by training exercise, improvements in cardiovascular disease in patients with T2DM and a reduction in urgent care visits and hospitalisations for preventable diabetes-related conditions. ${ }^{22-29}$ Additional studies involving weight loss of at least $10-15 \mathrm{~kg}$ have also achieved normalisation of blood glucose in people with short-duration T2DM. ${ }^{30}$

Therapeutic education is an ongoing process that aims to provide knowledge, skills and abilities necessary for the self-care of people with chronic diseases. According to the latest recommendations of the American Diabetes Association, everyone with diabetes should be part of education programmes from the moment of diagnosis. ${ }^{13}$ In countries such as the UK, there are educational programmes focused on the onset of T2DM (DESMOND Newly Diagnosed Programme). ${ }^{31}{ }^{32}$ Some clinical trials, both at the primary level and in managed care, have shown significant improvements in the goals of better control of T2DM, especially when nurses have a higher degree of autonomy. 152223

In Spain, there are prevention strategies ${ }^{33}$ and training initiatives among peers. ${ }^{34}$ Furthermore, the RedGEDAPS quality of care programme showed an improvement in the quality care indicators of T2DM. ${ }^{35}$ A study on T2DM onset, such as the Health Care and Therapeutic Education Programme for Newly Diagnosed Type 2 Diabetes pilot study, reported that the intervention was effective in terms of clinical results, educational results and patient satisfaction. ${ }^{25}$ Although there is favourable evidence, in the Spanish primary care setting, there are few specific sustainable programmes of structured education for people with a new diagnosis of T2DM, and they do not incorporate other prevalent diseases. Thus, it is evident that an ample room of improvement in the protocols used in the care of patients with a recent diabetes diagnosis exists, especially those with multiple comorbidities that can be easily adaptable to daily practice in the Spanish primary care setting.

\section{Aims and objectives}

The primary aim of this study was to determine the effectiveness of a systematic application of a comprehensive care protocol for people with a recent (less than 5 months) diagnosis of T2DM with associated comorbidities, on health-related quality of life (HRQoL), weight loss and HbAlc in primary healthcare centres when compared with usual care.

The secondary objective was to evaluate the effectiveness of the comprehensive care protocol compared with usual care on parameters as blood pressure, lipid profile, waist circumference, medication consumption, adherence to dietary recommendations, measures of physical activity, frequency of visits to the primary care centre and participant satisfaction.

\section{METHODS AND ANALYSIS \\ Study design}

A multicentre quantitative quasi-experimental design with a 1-year follow-up of patients with a recent diagnostic of T2DM (less than 5 months) with comorbidities (hypertension, dyslipidaemia and obesity) will be used to compare the effectiveness of a comprehensive care protocol. Selection of the centres with intervention group (IG) and comparison group (CG) will be randomised. A computerised random integer programme will be used to refer worksites to condition. Cluster randomisation of the centres will be adopted to increase the feasibility of roll-out in the organisational setting and minimise contamination across conditions. The IG will receive intensive education and support, and the $\mathrm{CG}$ will receive standard care. In the calculation of the sample, the cluster effect attributable to this design has been considered. The CG will know that they are not providing the intervention, although they do not know what it is. The outcome variables will be evaluated in both groups at baseline and after 3, 6 and 12 months of inclusion in the study (figure 1).

\section{Scope of the study}

The study will be developed in 10 primary care health centres of the same urban district in the city of Barcelona: Passeig Sant Joan, Carles I, Joanic, Vila de Gràcia, Sanllehy (coordinating centre), La Sagrera, Congrès, Encants, Camp de'lArpa and Guinardó. These 10 centres 


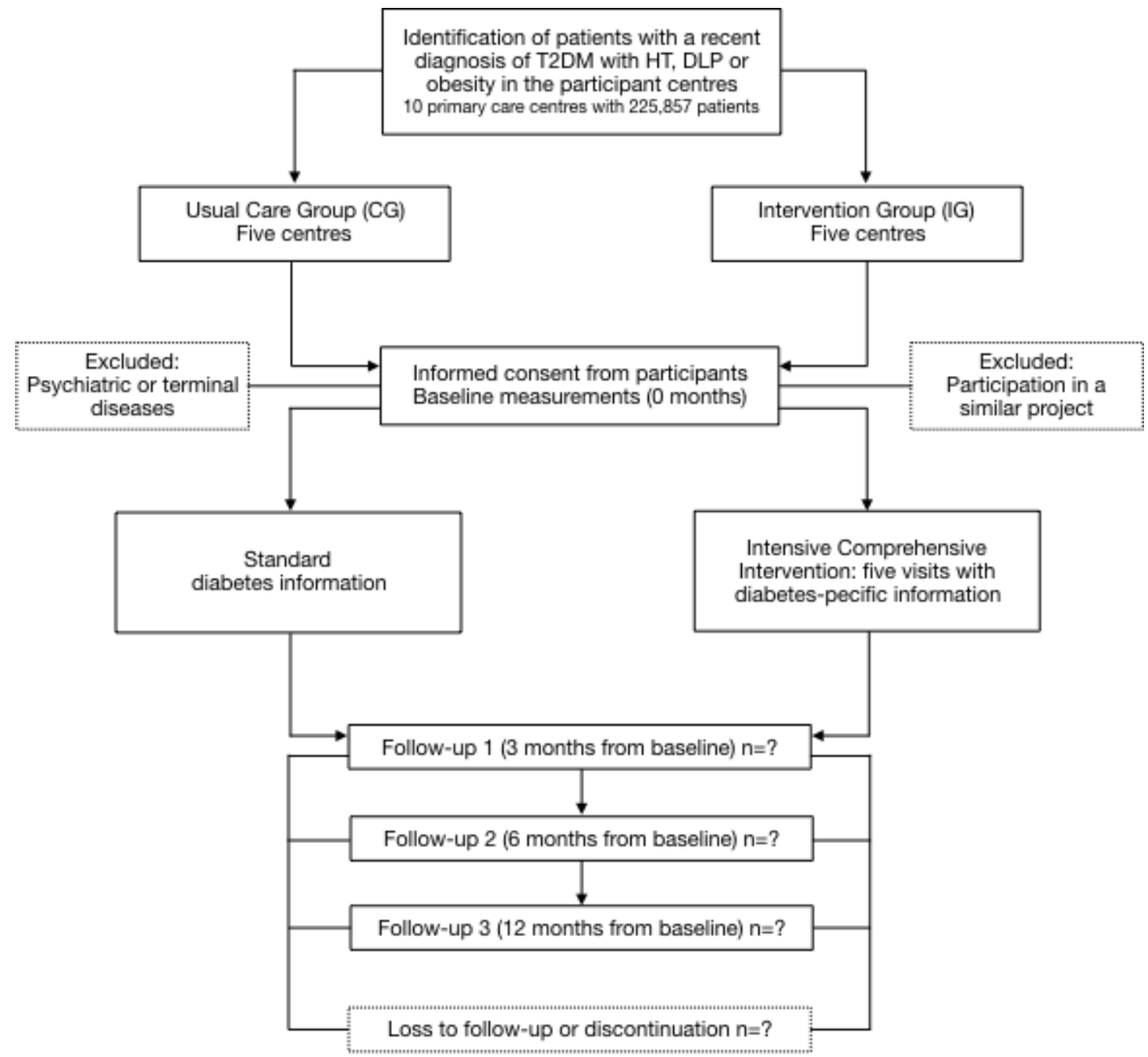

Figure 1 Flowchart of the intervention study. DLP, dyslipidaemia; HT, hypertension; T2DM, type 2 diabetes mellitus.

were selected because they belong to the reference area of the Hospital de la Santa Creu i Sant Pau and are managed by the Institut Català de la Salut. Centres run by independent healthcare providers were not included in the study. Five centres will be assigned to the IG, and the other five centres will be assigned to the CG by the research team.

\section{Subjects of the study}

The 10 participating primary care centres serve a population of 225857 , from which newly diagnosed T2DM cases (less than 5 months) that fulfil the inclusion criteria will be invited to participate; patients will be enrolled on their consent to participate. Electronic medical records from the Barcelona urban area report a T2DM prevalence of $7.02 \%$ in subjects over 18 years old, with up to $71.8 \%$ in clinical risk groups six or seven of low or medium severity, that is, significant chronic diseases in multiple organ systems with at least one (group 6) or two (group 7) dominant chronic diseases. ${ }^{36}$ The target population of patients with T2DM with comorbidities of low or medium severity presents $80 \%$ arterial hypertension, $13.5 \%$ ischaemic cardiovascular problems, $9.3 \%$ chronic obstructive pulmonary disease, $8.8 \%$ stroke, $8.1 \%$ renal insufficiency and $4.8 \%$ heart failure. Up to $70 \%$ of these subjects have two chronic conditions, and the remaining 30\% have three or more. Moreover, $41 \%$ are overweight and $42 \%$ are obese. The inclusion criteria are listed in table 1. All procedures and interventions for patient care are allowed except for participation in a similar protocol during the study.

\section{Recruitment process}

Eligible patient participants will be identified through the general practitioners and nurses of the primary care health centres. These professionals will be asked to refer patients who meet the inclusion criteria. Furthermore, the list of new diagnoses of T2DM with comorbidities provided by the information systems and healthcare quality unit (Unitat d'Avaluació, Sistemes d'Informació i Qualitat Assistencial, BASIQ) will be reviewed monthly. Patients who fulfil the inclusion criteria will be invited to participate in the study. Then, baseline data will be collected after obtaining written informed consent.

\section{Sample size}

The change in HRQoL score at 6 months from enrolment is the primary effectiveness endpoint. We will recruit and follow 123 participants per group for 2 years to detect a 


\begin{tabular}{|c|c|}
\hline Inclusion & Exclusion \\
\hline $\begin{array}{l}\text { Age between } 18 \text { and } 80 \text { years old. } \\
\text { A recent (less than } 5 \text { months) diagnosis of type } 2 \text { diabetes. } \\
\text { Two of the following comorbidities: previous diagnosed } \\
\text { hypertension ( } \geq 140 / \geq 90),{ }^{*} \text { dyslipidaemia (cholesterol }>200 \mathrm{mg} / \mathrm{dL} \\
\text { or } \geq 250 \mathrm{mg} / \mathrm{dL} \text { depending of CVRF) } \dagger \text { and/or obesity }(\mathrm{BMI} \geq 30 \text { ) } \\
\text { Adequate Catalan or Spanish literacy to complete the } \\
\text { assessments. }\end{array}$ & $\begin{array}{l}\text { Participation in another clinical trial or a similar } \\
\text { project. } \\
\text { Serious health problems: psychiatric or terminal } \\
\text { diseases. } \\
\text { Difficulties in maintaining participation for } 1 \text { year }\end{array}$ \\
\hline
\end{tabular}

${ }^{*}$ Clinical practice guidelines on arterial hypertension. ${ }^{45}$ †Clinical practice guidelines on cholesterol and coronary risk. ${ }^{46}$ $\mathrm{BMI}$, body mass index; CVRF, cardiovascular risk factor.

difference of $20 \%$ in HRQoL, accepting an alfa risk of 0.05 and a beta risk of 0.2 , and assuming a design effect of 1.2. Additionally, we took into account an estimated $10 \%$ participant loss and differences between the groups. The sample size was calculated based on the expected improvement of the HRQoL of the participants. It was assumed that $70 \%$ of participants who receive the intervention will improve his/her HRQoL and that only $50 \%$ of participants who receive standard care will improve. According to the diabetes incidence of 5.3/1000 patients/ year reported in primary healthcare centres in Barcelona city in 2018, a sufficiently large number of patients with a new diagnosis of T2DM will be available to fulfil the sample size. ${ }^{25}$

\section{Data collection and follow-up}

The primary endpoints are the change in HRQoL, glycaemic control (HbAlc) and weight loss at 6 and 12 months from baseline. The data collection will be carried out simultaneously through self-administered questionnaires (quality of life, diet and physical activity) and the measurement of biological variables in both groups, intervention and comparison, before initiating the intervention, after the intervention (3 months), and 6 and 12 months after the intervention. A specific evaluation of the knowledge acquired will not be carried out, but its incorporation into the patients' lives will be reflected in the improvement of diabetes control and their quality of life. Original study questionnaires will be entered and kept on file at the participating centre or the coordinating centre. All data will be recorded by a nurse in every centre, and monthly data with any identifying participant information removed will be sent to the coordinating centre. All investigators will be given access to the cleaned datasets. Recruitment and follow-up began in September 2019, and we expect to end in September 2021.

The following data will be collected from participants, when appropriate, using validated tools at baseline, after the intervention period ( 3 months), and at 6 and 12 months:

- EuroQol-5D (EQ-5D-3L) questionnaire. ${ }^{37}$ This is a generic tool used as a measure of HRQoL. This tool obtains information through five dimensions: mobility, self-care, usual activities, pain/discomfort and anxiety/depression. Each dimension has three levels (no problems, some problems and extreme problems). This scale can be converted to a unique index. The utility index of quality of life was also created based on previous studies. ${ }^{38} 39$ As a result of an intervention aimed at improving the control of chronic diseases and minimising their complications, the quality of life of the people served is a main outcome.

- Prevention with Mediterranean Diet (PREDIMED) questionnaire. ${ }^{40}$ This is a tool consisting of 14 items aimed at measuring adherence to the Mediterranean diet. This indicator will allow us to assess how knowledge has been incorporated by modifying life habits related to diet.

- International Physical Activity Questionnaire (IPAQ). ${ }^{41}$ IPAQ is an instrument for monitoring physical activity and inactivity. Data from the short IPAQ form will be summarised according to the physical activities recorded (walking, moderate and vigorous activities) and the estimated time spent sitting per week. Data will be used to estimate the total weekly physical activity by weighting the reported minutes per week within each activity category by a the metabolic equivalent energy expenditure estimate assigned to each category of activity. This indicator will allow us to assess the modification of lifestyle habits related to exercise.

- Satisfaction Questionnaire adapted from the questionnaire of the Catalan Health Service. This is a generic tool used as a measure of satisfaction level of participants regarding the attention, instruction and material received during the intervention. Participants will be asked to complete this questionnaire to measure their satisfaction level with the provided attention in the primary healthcare centres after their T2DM diagnosis.

Patient demographics such as age, gender, marital status (single/married/divorced/widowed), cohabitation status (living alone/living with someone else), educational level (illiterate/primary/secondary/university) and employment (currently working/unemployed/retired) will be collected. The patients' medical records will be reviewed 


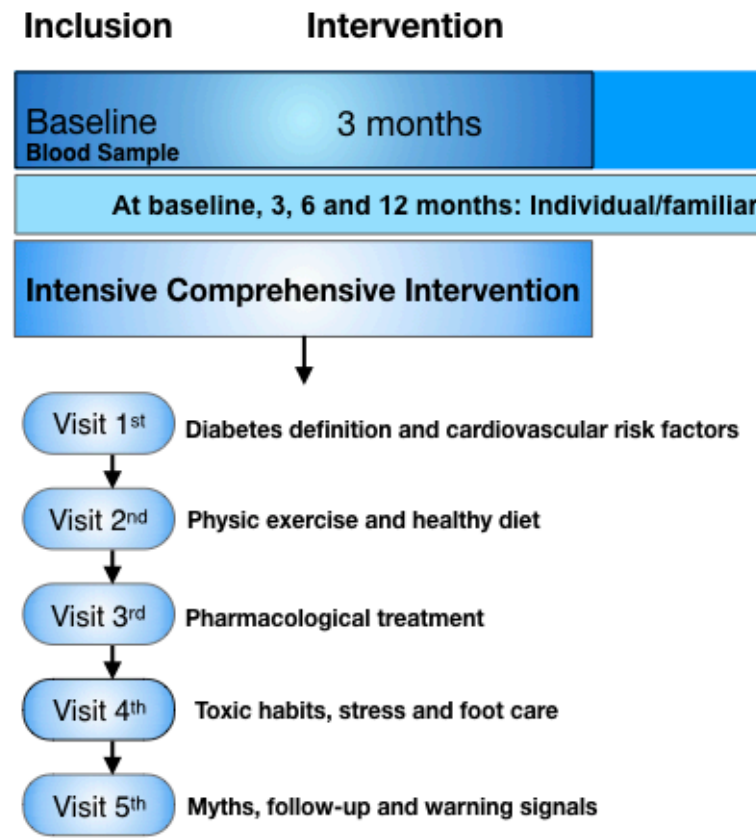

Figure 2 Structured intensive intervention and data collection.

to obtain clinical and medication histories. Further, chronic pathologies and their diagnosis dates, tobacco and alcohol consumption, and use of healthcare services in the last year will be collected at baseline and at the end of the study. We will also collect specific biological variables related to T2DM and associated comorbidities: HbA1c, weight, BMI, abdominal perimeter, blood pressure and cholesterol. All these variables will allow us to determine the clinical impact of this project.

\section{Intervention}

The intervention consists of an intensive health education protocol offered after the diagnosis of T2DM (less than 5 months) (see figure 2). It provides a triple action to address all the aspects that influence the good management of T2DM and a commitment to self-care:

- Knowledge: about the T2DM and associated comorbidities (offering the minimum needed but allowing providers to tailor it for each person), the warnings signs and the necessary monitoring tests.

- Abilities: how to perform self-care.

- Act/motivation: how to engage in self-care.

This comprehensive approach is developed sequentially in the primary care nurse office. Five individual intensive visits will be carried out with a weekly or biweekly frequency (according to the learning process) to obtain all the necessary information for comprehensive care and to transfer the knowledge and skills that are required for the proper management of the disease. The maximum time expected to complete these five visits is 3 months. During at least one of the visits, preferably the one that deals with dietary aspects, the patient will be asked to attend with her/his partner, child or cohabitants.

The five modules address the following:

\section{Follow-up}

1. What is diabetes? General concepts and cardiovascular risk factors.

2. Physical exercise and healthy diet.

3. Pharmacological treatment.

4. Toxic habits, stress and foot care.

5. Myths of diabetes: tracking tests and alarm signals.

The research team developed the support materials that contain the minimum contents that every patient newly diagnosed with T2DM and other chronic pathologies should know, based on a previous review of the recommendations of the clinical practice guidelines of reference. These support materials were approved by the hospital diabetes educators and family doctors in the area. A graphic designer was hired to give the materials an attractive and enticing design. The materials were validated with a group of patients who verified their utility and intelligibility. The support materials integrate all the agreed on minimum knowledge in a structured way and allow for the incorporation of cultural and emotional aspects. It is delivered to and discussed with the patient during the intervention. Once the five sessions finish, the patient will have consultation and reinforcement material that can be reconsulted when deemed appropriate.

In the IG group, once this first stage of higher intensity care has been completed, during which each patient must integrate the knowledge and skills necessary to assume his/her self-care and proper management of diabetes, the patients will continue their individual follow-up with their general practitioners, with the frequency established according to the associated comorbidities in each case as in the usual care group. The nurses of all centres will participate in two to four sessions to explain the study and the variables to be collected. Furthermore, the nurses of the centres in which the intervention will be carried out 
will undergo specific training. This training will consist of a detailed explanation of the intervention, the participant support materials will be reviewed and delivered, and a written guide specifically developed to unify the criteria when carrying out the therapeutic education will also be given to the nurses. The time calculated to recruit the individuals needed for the study and follow them is 2 years. Since the nurses in the study will be the patient's usual primary care nurse, we anticipate that the nurse's relationship with each participant will help promote patient permanence in the study. Participants who have missed a visit will be contacted by phone by the nurse to invite them to a new visit.

\section{Monitoring}

The monitoring committee will consist of four nurses from the research team. These nurses will remind monthly the nurse in charge of each primary care health centre to present reports of the data already collected, send the lists of newly diagnosed and pending data, and remind information regarding the intervention and data collection (figure 2). Preliminary extractions will be made to assess the evolution of the study and the quality of the information collected. The end of the trial will be when the required study sample is reached; we estimate that this will take 2 years. The centre nurses will be blinded for the results of the interim analyses at 3 and 6 months. Nurses from participating centres will send the possible adverse events to the research team, and participants can express any discomfort in the Satisfaction Questionnaire. Meetings with researchers will be held in the participating centres to promote knowledge, unify criteria and exchange information before the start of the study and every 6 months. The nurses of the participating centres will have at their disposal the telephone number and email of two researchers to answer any question that may arise during the recruitment and follow-up process. At least, monthly contacts and information exchange will be made between the nurses of each centre.

\section{Patient and public involvement}

No patients were involved in this study.

\section{Data analysis plan}

Initial data analysis will be performed to debug and validate the data entered into the dataset. Initially, baseline characteristics of both groups will be evaluated to assess homogeneity in age, comorbidities and exposure. To assess the comparability of baseline characteristics between the study groups, we will use a $\chi^{2}$ test or Fisher's exact test for nominal variables and Student's t-test or the Mann-Whitney test for continuous variables. The changes in main outcomes will also be assessed, by group, for the different periods, using a multilevel model, such as a linear mixed model, at baseline, 3, 6 and 12 months adjusted by the centre. Estimations will be adjusted by significant variables in univariate analyses and other clinically relevant factors. As a sensitivity analysis, unadjusted estimates will also be presented in different scenarios to assess the robustness of the results. Additionally, two subgroups will be evaluated to give robustness to the results: the intention-to-treat subgroup, which will consider all participants assigned to each group independently of whether they complete the entire protocol or not, and the 'per protocol' subgroup. The criterion for per protocol subgroup assignment will be completion of the entire protocol. The analysis will be carried out according to the statistical analysis plan. The $95 \%$ CIs and $p$ values will be computed with robust SEs to account for the cluster design sample, and statistical significance will be established as a p value of $<0.05$. Statistical analysis will be performed using SPSS software V.22.

\section{Ethics and dissemination}

Ethics approval for this study, including all sites, has been obtained from the Ethical Committee of Clinical Investigation of the IDIAP Jordi Gol (approval code: P13/118). All recruited patients will be informed by the nurses, verbally and in writing, about the objectives, methodology, tests and interventions they may receive if they participate in the study. They will be included if they grant their permission and sign the informed consent form (online supplementary file) in writing. This document will be written in a language (Spanish and Catalan) that the patients can understand. All participant information will be stored in locked file cabinets in areas with limited access. Local databases will be secured with password-protected access systems. Any modifications to the protocol will require a formal amendment to the protocol and acceptance by the ethical committee. We plan to publish the findings in peer-reviewed journals and share our findings at scientific conferences. The investigators will consider authorship following widely accepted criteria.

\section{Limitations of the study}

This protocol has some weaknesses. First, the allocation of the participants in the study will not be randomised. To avoid possible contamination of the staff who perform the intervention and those who provide standard care, every centre will have only one group of participants. Second, the grouping of the participants may be biassed if the homogeneity conditions are not met. These possible differences between groups have been taken into account for the calculation of the sample size. Third, the questionnaire that will be used to measure the HRQoL is not specific to people with diabetes. However, this questionnaire is extensively used in interventional studies involving patients with different diseases. ${ }^{43}{ }^{44}$ Fourth, we do not assess the behaviour change of health professionals who are responsible for delivering the intervention. Finally, acquired knowledge has not been evaluated as an outcome in this study; instead, we will evaluate the changes in lifestyles, which are an expression of the newly acquired knowledge during the intervention. 


\section{Author affiliations}

${ }^{1}$ Centre d'Atenció Primària Sanllehy, Gerència d'Àmbit d'Atenció Primària Barcelona Ciutat, Institut Català de la Salut, Barcelona, Spain

${ }^{2}$ Unitat de Suport a la Recerca Barcelona Ciutat, Fundació Institut Universitari per a la recerca en Atenció Primària de Salut Jordi Gol i Gurina (IDIAP Jordi Gol), Barcelona, Spain

${ }^{3}$ Gerència Territorial de Barcelona Ciutat, Institut Català de la Salut, Barcelona, Spain

${ }^{4}$ Centre d'Atenció Primària Sagrera, Gerència d'Àmbit d'Atenció Primària Barcelona Ciutat, Institut Català de la Salut, Barcelona, Spain

${ }^{5}$ Unidad de Evaluación, Sistemas de Información y Calidad, Institut Català de la Salut, Barcelona, Spain

${ }^{6}$ DAP-Cat Group, Unitat de Suport a la Recerca Barcelona, Fundació Institut Universitari per a la Recerca a I'Atenció Primària de Salut Jordi Gol i Gurina (IDIAPJGol), Barcelona, Spain

${ }^{7}$ Department of Endocrinology and Nutrition, Hospital de la Santa Creu i Sant Pau \& Institut d'Investigació Biomédica Sant Pau (IIB Sant Pau) \& Centre for Biomedical Research on Diabetes and Associated Metabolic Diseases (CIBERDEM), Barcelona, Spain

Acknowledgements CIBER of Diabetes and Associated Metabolic Diseases is an initiative from Instituto de Salud Carlos III (Plan Nacional de I+D+l and Fondo Europeo de Desarrollo Regional). The research team thanks the participant and professional advisers who generously shared their time, experience and knowledge for the purposes of this project.

Contributors $\mathrm{CL}$ conceived of the study. $\mathrm{CL}$ and VL initiated the study design, and $\mathrm{EB}, \mathrm{CD}, \mathrm{RA}, \mathrm{AG}$ and EC helped with implementation. CL, VL, JLdVG, CD and RA are grant holders. JLdVG provided statistical expertise in clinical trial design and will be conducting the primary statistical analysis. All authors contributed to refinement of the study protocol and approved the final manuscript.

Funding This study has been funded in part by the Nursing and Society Foundation as part of the Nurse Research Projects Grants PR-042/16. This funding source had no role in the design of this study and will not have any role during its execution, analyses and data interpretation, or in making the decision to submit its results for publication.

Competing interests None declared.

Patient and public involvement Patients and/or the public were not involved in the design, or conduct, or reporting, or dissemination plans of this research.

Patient consent for publication Not required.

Ethics approval The Ethical Committee of Clinical Investigation of the IDIAP Jordi Gol has approved of this protocol (approval code: P13/118).

Provenance and peer review Not commissioned; externally peer reviewed.

Open access This is an open access article distributed in accordance with the Creative Commons Attribution Non Commercial (CC BY-NC 4.0) license, which permits others to distribute, remix, adapt, build upon this work non-commercially, and license their derivative works on different terms, provided the original work is properly cited, appropriate credit is given, any changes made indicated, and the use is non-commercial. See: http://creativecommons.org/licenses/by-nc/4.0/.

ORCID iDs

Carolina Lapena http://orcid.org/0000-0003-0040-6505

Esmeralda Castelblanco http://orcid.org/0000-0002-2061-6270

\section{REFERENCES}

1 Cho NH, Shaw JE, Karuranga S, et al. IDF diabetes atlas: global estimates of diabetes prevalence for 2017 and projections for 2045. Diabetes Res Clin Pract 2018;138:271-81.

2 Soriguer F, Goday A, Bosch-Comas A, et al. Prevalence of diabetes mellitus and impaired glucose regulation in Spain: the Di@bet.es Study. Diabetologia 2012;55:88-93.

3 Valdés S, Rojo-Martínez G, Soriguer F. [Evolution of prevalence of type 2 diabetes in adult Spanish population]. Med Clin 2007;129:352-5.

4 Crespo C, Brosa M, Soria-Juan A, et al. Direct cost of diabetes mellitus and its complications in Spain (SECCAID study: Spain estimated cost Ciberdem-Cabimer in diabetes. Av Diabetol 2013;29:182-9.
5 Patel A, MacMahon S, et al, ADVANCE Collaborative Group, ADVANCE Collaborative Group. Intensive blood glucose control and vascular outcomes in patients with type 2 diabetes. N Engl J Med 2008;358:2560-72.

6 Gerstein HC, Miller ME, Byington RP, et al. Action to control cardiovascular risk in diabetes Study Group. Effects of intensive glucose lowering in type 2 diabetes. N Engl J Med2008;358:2545-59.

7 Lipska KJ, Krumholz H, Soones T, et al. Polypharmacy in the aging patient: a review of glycemic control in older adults with type 2 diabetes. JAMA 2016;315:1034-45.

8 Krans HMJ, Porta M, Keen H. Diabetes care and research in Europe. The Saint Vincent Declaration action programme. Ginebra: WHO Regional Office for Europe, 1992.

9 Cano JF, Franch J, Mata M, et al. Guide for the treatment of type 2 diabetes in primary care. 4ª edición. Madrid: Harcourt Ed, 2004.

10 IDF Clinical Guidelines Task Force. Global guideline for type 2 diabetes: recommendations for standard, comprehensive, and minimal care. Diabet Med 2006;23:579-93.

11 Rydén L, Grant P, Anker SD, et al. Guidelines on diabetes, prediabetes and cardiovascular diseases developed in collaboration with the EASD. Eur Heart $J$ 2013;39:3035-87.

12 American Diabetes Association. Standards of medical care in diabetes--2013. Diabetes Care 2013;36 Suppl 1:S11-66.

13 Association AD. Standars of medical care in diabetes 2017. Diabetes Care 2017; 40:533-43.

14 Lean ME, Leslie WS, Barnes AC, et al. Primary care-led weight management for remission of type 2 diabetes (direct): an open-label, cluster-randomised trial. Lancet 2018;391:541-51.

15 Holman RR, Paul SK, Bethel MA, et al. 10-Year follow-up of intensive glucose control in type 2 diabetes. $N$ Engl J Med Overseas Ed 2008;359:1577-89.

16 Liebl A, Mata M, Eschwège E. Evaluation of risk factors for development of complications in type II diabetes in Europe. Diabetologia 2002;45:S23-8.

17 de la Calle H, Costa A, Díez-Espino J, et al. [Evaluation on the compliance of the metabolic control aims in outpatients with type 2 diabetes mellitus in Spain. The TranSTAR study]. Med Clin 2003;120:446-50.

18 López B, García-Mayor R, Puig-Domingo M, et al. Pathological characteristics of patients with diabetes mellitus TipE 2, in Spanish primary care. Rev Clin Esp 2004;1:18-24.

19 Alvarez-Sala LA, Suárez C, Mantilla T, et al. [PREVENCAT study: control of cardiovascular risk in primary care]. Med Clin 2005;124:406-10.

20 Orozco-Beltrán D, Gil-Guillen VF, Quirce F, et al. Control of diabetes and cardiovascular risk factors in patients with type 2 diabetes in primary care. the gap between guidelines and reality in Spain. Int $J$ Clin Pract 2007;61:909-15.

21 Mengual L, Roura P, Serra M, et al. Multifactorial control and treatment intensity of type-2 diabetes in primary care settings in Catalonia. Cardiovasc Diabetol 2010;9:14.

22 Glechner A, Keuchel L, Affengruber L, et al. Effects of lifestyle changes on adults with prediabetes: a systematic review and metaanalysis. Prim Care Diabetes 2018;12:393-408.

23 Taylor CB, Miller NH, Reilly KR, et al. Evaluation of a nursecare management system to improve outcomes in patients with complicated diabetes. Diabetes Care 2003;26:1058-63.

24 Costa-Pinel B, Mestre-Miravet S, Barrio-Torrell F, et al. Implementation of the DP-TRANSFERS project in Catalonia: a translational method to improve diabetes screening and prevention in primary care. PLoS One 2018;13:e0194005.

25 Colungo $\mathrm{C}$, Liroz M, Jansà M, et al. Health care and therapeutic education program for newly diagnosed type 2 diabetes: a new approach in primary care. Endocrinol Diabetes Nutr 2018;65:486-99.

26 Chen L, Pei J-H, Kuang J, et al. Effect of lifestyle intervention in patients with type 2 diabetes: a meta-analysis. Metabolism 2015;64:338-47.

27 Davidson MB, Ansari A, Karlan VJ. Effect of a nurse-directed diabetes disease management program on urgent care/emergency room visits and hospitalizations in a minority population. Diabetes Care 2007;30:224-7.

28 Boulé NG, Haddad E, Kenny GP, et al. Effects of exercise on glycemic control and body mass in type 2 diabetes mellitus: a metaanalysis of controlled clinical trials. JAMA 2001;286:1218-27.

29 Salas-Salvadó J, Bulló M, Babio N, et al. Reduction in the incidence of type 2 diabetes with the Mediterranean diet: results of the PREDIMED-Reus nutrition intervention randomized trial. Diabetes Care 2011;34:14-19.

30 Sjöström L, Peltonen M, Jacobson P, et al. Association of bariatric surgery with long-term remission of type 2 diabetes and 
with microvascular and macrovascular complications. JAMA 2014;311:2297-304.

31 Davies MJ, Heller S, Skinner TC, et al. On behalf of the DESMOND collaborative. effectiveness of the diabetes education for ongoing and newly diagnosed (DESMOND) program for people with newly diagnosed type 2 diabetes: cluster randomized controlled trial. BMJ 2008;336:491-5

32 Ockleford E, Shaw RL, Willars J, et al. Education and selfmanagement for people newly diagnosed with type 2 diabetes: a qualitative study of patients' views. Chronic IIIn 2008;4:28-37.

33 Sagarra R, Costa B, Cabré JJ, et al. El Grupo de Investigación DEPLAN-CAT/PREDICE. lifestyle interventions for diabetes mellitus type 2 prevention. Rev Clin Esp 2014;214:59-68.

34 Nuño-Solinis R, Rodríguez-Pereira C, Piñera-Elorriaga K, et al. [Panorama of self-management initiatives in Spain]. Gac Sanit 2013;27:332-7.

35 Mata-Cases M, Roura-Olmeda P, Berengué-Iglesias M, et al. Fifteen years of continuous improvement of quality care of type 2 diabetes mellitus in primary care in Catalonia, Spain. Int $J$ Clin Pract 2012;66:289-98.

36 Hughes JS, Averill RF, Eisenhandler J, et al. Clinical risk groups (CRGs): a classification system for risk-adjusted capitation-based payment and health care management. Med Care 2004;42:81-90.

37 Brooks R. EuroQol: the current state of play. Health Policy 1996;37:53-72.

38 Rosser RM, Watts VC. The measurement of hospital output. Int J Epidemiol 1972;1:361-8.
39 Hunt SM, McEwen J, McKenna SP. Measuring health status: a new tool for clinicians and epidemiologists. J $R$ Coll Gen Pract 1985;35:185-8.

40 Estruch R, Martínez-González MA, Corella D, et al. Effects of a Mediterranean-style diet on cardiovascular risk factors: a randomized trial. Ann Intern Med 2006;145:1-11.

41 Craig CL, Marshall AL, Sjöström M, et al. International physical activity questionnaire: 12 -country reliability and validity. Med Sci Sports Exerc 2003;35:1381-95.

42 Román-Vinas B, Ribas-Barba L, Ngoa J, et al. Validity of the International physical activity questionnaire in the Catalan population (Spain). Gac Sanit 2013;3:254-7.

43 Yang J, Jit M, Zheng Y, et al. The impact of influenza on the health related quality of life in China: an EQ-5D survey. BMC Infect Dis 2017;17:686

44 Vermeulen KM, Jansen DEMC, Buskens E, et al. Serious child and adolescent behaviour disorders; a valuation study by professionals, youth and parents. BMC Psychiatry 2017;17:208.

45 Coll de Tuero G, de la Sierra Iserte A, Salleras Marcó N, et al. Arterial hypertension [En línia] Barcelona: Institut Catal de la Salut, 2012. Clinical practice guidelines and teaching material, núm. 6, 2012. Available: http://www.gencat.cat/ics/professionals/guies/hipertensio/ hipertensio.htm

46 Díez B, Colomer JM; Barcelo, Misol E; Ciurana, et al. Cholesterol and coronary risk [En línia] Barcelona: Institut Catal de la Salut, 2009. Clinical practice guidelines and teaching material, núm. 1, 2009. Available: http://www.gencat.cat/ics/professionals/guies/colesterol/ colesterol.htm 